\title{
Metamorphosis of Nuclear Medicine
}

\section{Issa Loutfi}

Departments of Nuclear Medicine, Kuwait University, P. O. Box 24923, Safat 13110, Kuwait

This special issue of the International Journal of Radiology and Medical Imaging on nuclear medicine in diagnosis and treatment comes at a time of rapid evolution of applications involving the use of unsealed radioactive sources in the management of various disease conditions, which is the main subject matter of nuclear medicine.

Over the last few years only, many tests have been added to routine nuclear medicine practice especially in its diagnostic imaging arm. The most prominent battery of new tests involves the adaptation of positron emission tomography (PET) from an elaborate research tool to an essential aide in diagnosis, especially in oncology. A whole discipline has emerged aiming at studying specific biochemical events in vivo in what is now known as Molecular Imaging. In addition, refinement in the imaging instrumentation, especially the hybrid function-structure combination such as PET/CT, has enabled nuclear medicine to reach unprecedented levels of diagnostic accuracy and clinical usefulness [1].

The therapy arm of nuclear medicine has attracted much interest to become a fertile area of research at the present time. A few clinical applications have seen light and have already benefited patients who have been refractory to more conventional types of therapy. The most interesting concept in the treatment arena is Theranostics [2] in which a diagnostic-therapeutic radionuclide pair is used to target a biological process, first for detection and second for destruction. Various candidate pairs for such a strategy are being tested and some have been released for clinical use from ${ }^{123} \mathrm{I}^{-131} \mathrm{I}$ for differentiated thyroid cancer to ${ }^{68} \mathrm{Ga}-{ }^{177} \mathrm{Lu}$ PSMA for prostate cancer.

- Based on the overview of the current scope of nuclear medicine, the contents of the special issue are divided in two parts. The first part is for the current status and new developments in the basic science aspects of nuclear medicine and includes:

- Radiotracers and development of molecular imaging probes especially for components of biochemical pathways in pathological conditions. 18Fluoro deoxyglucose (FDG) has been successfully used to map glucose metabolism in tumors and infections. Many more potential candidates for molecular imaging are being synthesized including radiolabeled hormones, growth receptors, hypoxia markers, etc.

- Imaging instrumentation and protocols: the advent of hybrid anatomy-physiology approach to imaging by combination of structure-based modalities such as CT and function-based detectors such as the PET scanner or the scintillation camera has provided a solution to the often encountered dilemma of localization of areas of abnormal uptake on the nuclear medicine scan and gave a way to resolve the technical issue of attenuation correction in tomographic imaging. More hybrid combinations are currently being established for routine use such as PET-MRI.

- Radiation risks: The use of the newer radiotracers and especially the addition of structural imaging such as CT to nuclear medicine procedures have resulted in a modest increase in the radiation dose to the patient. These issues had to be addressed to ensure safe and ethical use of the techniques. The concepts of Image Wisely and Image Gently have been put forward especially for imaging in pediatrics.

\section{Publication History:}

Received: August 26, 2015

Accepted: September 22, 2015

Published: September 24, 2015

\section{Keywords:}

Nuclear medicine, Hybrid imaging, Molecular imaging, Cancer, Theranostics

- Practical and logistical matters: The current health care environment is that for cutting cost and providing efficient service to patients. As a rapidly developing field with many innovations, nuclear medicine has to update itself more frequently especially keeping up with new radiopharmaceuticals, instrumentation, training of personnel and competition from other imaging modalities. In addition, a continuous quality assurance program is mandatory to provide trusted and accurate contribution to patient care.

The second part of the special issue is for the clinical applications, both diagnostic and therapeutic, of nuclear medicine and identifying its role in various disease conditions and includes:

- Nuclear cardiology: perfusion, function, viability, and sympathetic innervations. Cardiac applications remain the most common in the majority of nuclear medicine departments. The provision of accurate diagnosis of coronary artery disease is the main objective in the evaluation of various patient populations especially those with diabetes, hypertension, and others. Technical issues such as hybrid imaging and the study of myocardial viability and in some cases sympathetic innervations in patients with heart failure using 123I MIBG can add valuable information that could be useful for patient management [3].

- Diagnosis and monitoring of cancer: Currently, the management of patients with malignant tumors of all types relies heavily on a multiplicity of nuclear medicine tests, especially those involving PET. The gauging of tumor response to radio- or chemotherapy is an area of thorough investigation to set up protocols for tumor monitoring and follow up [4]. An example of such elegant research is the use of FDG PET in the management of lymphoma.

- Detection and monitoring of inflammation: The introduction and testing of new radiotracers such as FDG [5] and protocols for special pathological conditions such as bone, white cell and bone marrow scans with SPECT/CT imaging in the evaluation of the diabetic foot are being set up and refined.

- Urological applications in evaluation of obstruction and infections. Also, a special interest in the evaluation of prostate cancer has been particularly pursued.

*Corresponding Author: Dr. Issa Loutfi, Departments of Nuclear Medicine, Kuwait University, P. O. Box24923, Safat 13110, Kuwait, E-mail: loutfi@hsc.edu.kw

Citation: Loutfi I (2015) Metamorphosis of Nuclear Medicine. Int J Radiol Med Imag 1: 104. doi: http://dx.doi.org/10.15344/ijrmi/2015/104

Copyright: ( 2015 Loutfi. This is an open-access article distributed under the terms of the Creative Commons Attribution License, which permits unrestricted use, distribution, and reproduction in any medium, provided the original author and source are credited. 
- $\quad$ Endocrine: thyroid, parathyroid, adrenal. Thyroid applications have always been a mainstay of nuclear medicine practice. Issues regarding management of thyroid cancers have emerged with more detection of tumors and outcome analysis of low versus high risk cancers. Parathyroid imaging has benefited from the use of SPECT/CT while the adrenals have been targeted more specifically with radiotracers for medullary or cortical function.

- CNS: dementia, Parkinsonism. Neurological investigations using nuclear medicine procedures have developed tremendously in the last few years with a growing interest for evaluation of dementia, epilepsy and movement disorders. Amyloid plaque imaging and neuroreceptor quantification have been studied extensively to provide an objective evaluation of these conditions, which would help in their clinical management.

- Musculoskeletal: children and adults: The role of various imaging procedures for assessment of pathological conditions of the skeleton including infections and tumors has been the subject of constant evolution and debate. The impact of nuclear medicine procedures starting with the standard bone scan and ending with sodium fluoride PET/CT scan has been recognized for conditions affecting the skeleton such as osteomyelitis and bone metastasis [6]. Currently, strategies for imaging have been postulated depending on the clinical presentation and suspicion of a certain pathological process. The delivered radiation dose has been a hot issue of debate in pediatrics.

- Radionuclide therapy: Theranostics. Targeting radiation specifically to a certain site in the body has made radionuclide therapy very attractive in clinical practice. An even more interesting concept is to be able to use an imaging tool to detect pathology by using a suitable gamma-ray emitting radionuclide then using the same tool for therapy by substituting the gammaemitter with a particulate emitter (beta or alpha particles). This approach, known as theranostics, has been the subject of extensive research in recent years for diagnosis and therapy of tumors [2]. Radionuclide therapy is projected to grow dramatically in the near future. There are already facilities in some nuclear medicine departments to provide radionuclide treatment; the most notable of them are for neuroendocrine tumors.

It is hoped that the special issue on Nuclear Medicine in Diagnosis and Treatment would benefit the workers in the field and would be interesting to readers at large.

\section{Competing Interests}

The authors have declared that no competing interests exist.

\section{References}

1. Patton JA, Townsend DW, Hutton BF (2009) Hybrid imaging technology: from dreams and vision to clinical devices. Semin Nucl Med 39: 247-263.

2. DeNardo GL, DeNardo SJ (2012) Concepts, consequences, and implications of theranosis. Semin Nucl Med 42: 147-150.

3. Travin MI (2014) Cardiac radionuclide imaging to assess patients with heart failure. Semin Nucl Med 44: 294-313.

4. Mortimer JE, Dehdashti F, Siegel BA, Katzenellenbogen JA, Fracasso P, et al. (1996) Positron emission tomography with 2-[18F] Fluoro-2-deoxyD-glucose and 16alpha-[18F] fluoro-17beta-estradiol in breast cancer: correlation with estrogen receptor status and response to systemic therapy. Clin Cancer Res 2: 933-939.

5. Bleeker-Rovers CP, Corstens FH, Van Der Meer JW, Oyen WJ (2004) Fever of unknown origin: prospective comparison of diagnostic value of (18)F-FDG PET and (111)In-granulocyte scintigraphy. Eur J Nucl Med Mol Imaging 31: 1342-1343.
6. Grant FD, Fahey FH, Packard AB, Davis RT, Alavi A, et al. (2008) Skeletal PET with 18F-fluoride: applying new technology to an old tracer. J Nucl Med 49: 68-78.

\section{This article was originally published in a special issue:}

Nuclear Medicine in Diagnosis and Treatment

Handled by Editor(s):

Dr. Issa Loutfi

Departments of Nuclear Medicine

Kuwait University and Mubarak Al Kabeer Hospital

Kuwait 